\title{
Gavial: Programming the web with multi-tier FRP
}

\author{
Bob Reynders ${ }^{\mathrm{a}}$, Frank Piessens ${ }^{\mathrm{b}}$, and Dominique Devriese ${ }^{\mathrm{c}}$ \\ a Chonnam National University \\ b imec - DistriNet - KU Leuven \\ c Vrije Universiteit Brussel
}

Abstract Web applications are inherently distributed, and not just because their client and server counterparts run on networked systems. Web applications are written in multiple programming languages and as multiple programs: the server and client programs. In an effort to lower the complexity of the web, multi-tier programming was proposed. In multi-tier programming languages the language and its tooling give support to create web applications as a whole, one program is written in one language.

Web applications are also inherently asynchronous. On the server, they constantly process several client requests and in the browser they constantly have to react to input, be it from the user or a server. A technique that can be applied to make such programs easier to understand is functional reactive programming. A functional programming model that models an interactive program as compositions between two primitives, behaviors and events.

Developing web applications requires dealing with their distributed nature and the natural asynchronicity of user input and network communication. For facilitating this, different researchers have explored the combination of a multi-tier programming language and functional reactive programming. However, existing proposals take his approach only part of the way. Some parts of the application remain imperative, do not consider network traffic overhead of incremental data, compatibility with common APIs like XMLHttpRequest etc., or they do not consider glitches across network communication (or do but introduce locking or overhead)

In this paper, we present Gavial: a design and practical implementation of multi-tier FRP that allows constructing a web application as a functionally reactive program. We propose a novel integration of existing techniques such as solutions to bootstrapping web applications, recursive behaviors for web interfaces, asynchronous FRP and incremental behaviors. As well as novel support for tiered glitch freedom, a compromise between performance and glitch-free evaluation of the distributed FRP program, and a three-tiered system suitable for request-response-style applications as well as user-to-user applications. Gavial automatically runs in request-response (XMLHttpRequest) mode or in websocket mode.

After applying a number of old and new ideas to create a practical multi-tier FRP implementation in Gavial, we demonstrate it by building an example and show that it can in fact deal realistically with important practical aspects of building web applications. At the same time, we retain the declarative nature of FRP, where behaviors and events have an intuitive, compositional semantics and a clear dependency structure.

ACM CCS 2012

- Software and its engineering $\rightarrow$ Functional languages; Data flow languages; Domain specific languages; Keywords Functional reactive programming (FRP), multi-tier programming languages

\section{The Art, Science, and Engineering of Programming}

Submitted May 27, 2019

Published February 17, 2020

DoI 10.22152/programming-journal.org/2020/4/6 


\section{Gavial: Programming the web with multi-tier FRP}

\section{Introduction}

Developing web applications requires dealing with the specificities of the web. This includes the distributed nature of applications, partly executing on the client (i.e. the user's browser), partly on the server, and the different parts communicating over APIs like WebSockets or XMLHttpRequests. Additionally, user input and client-server communication on the web are both naturally asynchronous. These characteristics of web applications have led researchers to design programming languages or frameworks tailored to their development.

Two ideas to cope with the web's distributed and asynchronous nature are multi-tier programming languages and functional reactive programming. Multi-tier languages (see among others: $[8,19,29]$ ) allow both client and server parts of a web application to be written in a single codebase - offering a joint semantics and allowing cross-tier abstractions. On the other hand, functional reactive programming [12] is an alternative programming model that facilitates development of asynchronous applications and reasoning about their behavior. Instead of using side-effecting callbacks, FRP programs are constructed by composing behaviors and events: components representing timedependent values.

Combinations of FRP with multi-tier programming have been explored in the past. Multi-tier FRP has been presented with Scala in [25]. They provide .to(Client/Server) on events and behaviors. Eliom [23] provides a client/server reactive abstraction (since v5.0). ${ }^{\mathrm{I}}$ They also provide a signal and have similar tier-crossing primitives. These approaches conveniently connect multiple FRP programs across tiers, however they do not provide a unified programming experience. Programmers have to pay close attention not to get partial propagation (glitches) throughout the distributed FRP program in order to maintain consistency — something reactive programming typically takes care of.

Distributed reactive programming languages [II, I6, I8] have distributed consistency guarantees and allow distributed FRP propagation without observable partial propagations (glitches). ScalaLoci [35] in particular - a general purpose multi-tier extension of Scala which allows programmers to define their own distribution scheme supports tier-crossing primitives with distributed consistency guarantees. These guarantees are gained through a compromise of using global coordination or increasing message count or size.

The main contribution of this paper is Gavial: a design and implementation of a multi-tier FRP framework that allows constructing a web application as a functional reactive program: GUI definitions, request/response-style web apps as well as collaborative web apps such as video games are compositions of FRP primitives. We achieve this by incorporating (I) existing ideas: automatic bootstrapping [25], asynchronous FRP [9], recursive behaviors for building web interfaces [26] and incremental behaviors [24] and (2) introducing novel ideas: a form of glitch minimization across network communication called tiered glitch freedom (section 3.6), the three-tier structure of

\footnotetext{
${ }^{\mathrm{I}}$ https://opam.ocaml.org/packages/eliom/eliom.5.0.0/
} 




Figure 1 CircleRoyale

our behaviors and events (section 3.2), novel support for using XMLHttpRequests (instead of WebSockets) in the absence of server-initiated tier-crossing (section 3.7), and an implementation reusing existing Scala infrastructure and library eco-system (section 4). Gavial shows that multi-tier FRP for the web requires only thin abstractions on top of proven technologies. At the same time, we retain the declarative nature of FRP, where behaviors and events have an intuitive, compositional semantics and a clear dependency structure.

Outline First, we gradually introduce Gavial and its features by incrementally developing a game CircleRoyale (section 2). Next, we provide more details on Gavial APIs (section 3) and our implementation (section 4). We discuss related work in section 5 and conclude in section 6 .

\section{Multi-tier FRP by Example}

Gavial is an embedded domain specific language in Scala (JVM) and Scala.JS (a mature compiler targeting JavaScript). We introduce it using "CircleRoyale": a small game shown in figure I. It is inspired by a trend of simple online multiplayer games such as agar.io where players battle each other on a large playing field with minimal controls. In CircleRoyale, players (shown as circles) continuously move around in the direction of the mouse cursor. They can start attacks by hitting space, spawning a larger flashing circle around the player. It remains active for 2 seconds and then cannot be used for 3 seconds (the cooldown period). The game ends when the player is hit by an attack and the end score is the time spent alive.

In this chapter, we demonstrate this by gradually implementing CircleRoyale, introducing and highlighting features of Gavial along the way. We start from a simple single-player single-tier UI, and make small, local and understandable changes towards the end result. All code examples are valid Scala and actually running code. Example code is shown inline and detailed API information in captioned listings. We encourage the reader to try out Gavial on http://tzbob.be/gavial, only one command is required to setup a basic project and start the tutorial . Both the inline example (in stages) and the CircleRoyale game are available online: https://github.com/tzbob/circleroyale. 


\section{Gavial: Programming the web with multi-tier FRP}

Listing 1 Event and DBehavior

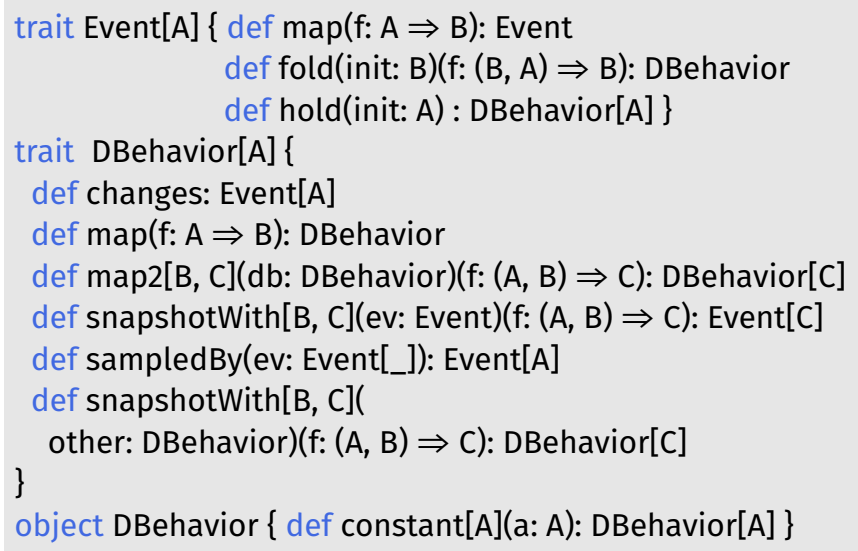

Figure 2 FRP Primitives

\subsection{Client Prototype}

We start with a working solo-player version of CircleRoyale in which a player can move around and start attacks but there are no opponents.

Functional Reactive Programming User controls are modeled as direction and attacking:

val attacking: DBehavior ${ }^{C}[$ Boolean $]=--$ cut --

val direction: DBehavior $[$ [Vec2D] = -- cut --

To understand this, let us clarify some terminology. Gavial offers a flavor of FRP that contains: events, behaviors, discrete behaviors and incremental behaviors which we discuss later on. As depicted in figure 2, events are streams of timestamped values and behaviors are time-varying values. Discrete behaviors only change at discrete times, which behave as right continuous step functions. For now, we use only Event and DBehavior, the superscript ${ }^{C}$ refers to computations on the client (see below for other tiers). Events and behaviors have the API shown in listing I.

Events can be mapped over with a function or be folded into discrete behaviors. Such folded discrete behaviors start out with the given initial value and "step" to a new value when the event fires, combining the event's value and the behavior's previous value using $\mathrm{f}$. hold is like fold but simply stores the last event value seen.

Behaviors can be combined using map2 and can be read at the rate of an event using snapshotWith (using a function to combine the values). sampledBy is like snapshotWith but ignores the value of event ev. A snapshotWith for discrete behaviors works like map2 but the resulting behavior changes only when other does. 
Listing 2 Delayed DBehavior

object DBehavior $\{$ def delayed[A](db: $\Rightarrow$ DBehavior[A]): Behavior $[A]\}$

Returning to CircleRoyale, a type Player represents a player's position, and whether he is alive, attacking or dead. The svg method produces the player's circle and optionally the attacking circle as SVG elements.

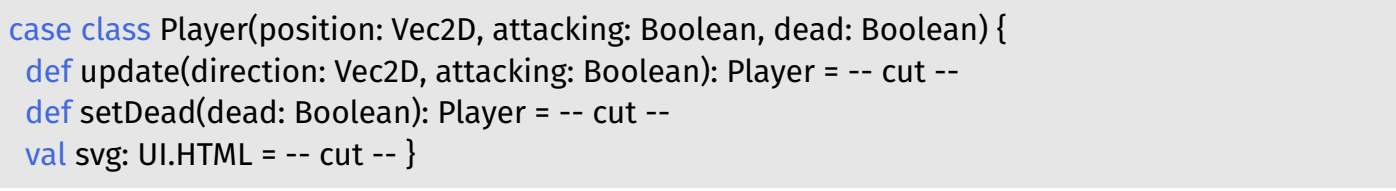

We define the player's state over time, by tupling the user controls (direction and whether an attack should be started) using map2 ${ }^{2}$ and then folding these changes starting from a default state. For this example we simplify a bit and allow players to attack whenever and for however long they want.

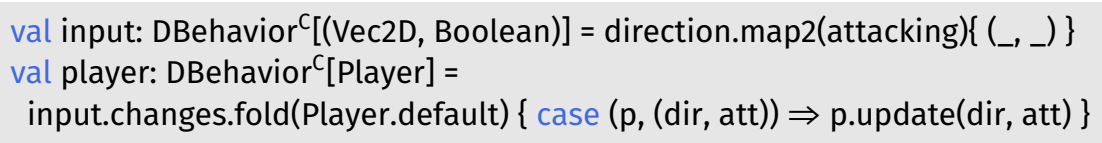

Throttling the update rate The user input in direction is based on mouse movement and updates very frequently, so for efficiency, we change player to throttle the rate. This is easy to do in Gavial using IntervalCycle (an abstraction around JavaScript's setInterval). We sample input by an event time which fires at Io $\mathrm{Hz}$ and update player to use this throttled input instead.

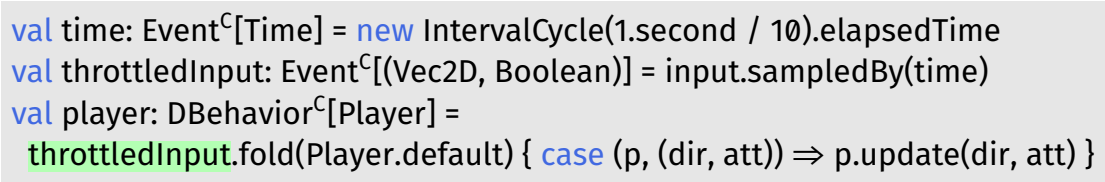

Recursive Behavior As pointed out before [26], user interfaces are often inherently recursive. For example, in CircleRoyale the future direction of a player is relative to its current position, which is itself determined by the direction of movement. Our flavor of FRP permits explicit recursive definitions (in Scala) through .delayed as shown in listing 2.

A delayed behavior will have the same step function as the original behavior but it is left continuous instead of being right continuous as in figure 2. In other words, the delayed version of a discrete behavior keeps the old value for an instant longer when a change occurs. Note that the $\mathrm{db}$ parameter is passed by-name and is not evaluated immediately, this Scala feature (and because of the implementation of delayed that makes use of it) makes it possible to make forward references to a behavior and makes self-recursive definitions possible. We use this particularly for defining direction.

\footnotetext{
$\left.\overline{{ }^{2}\left(\_,-\right.}\right)$is a Scala anonymous function that combines two arguments into a pair.
} 


\section{Gavial: Programming the web with multi-tier FRP}

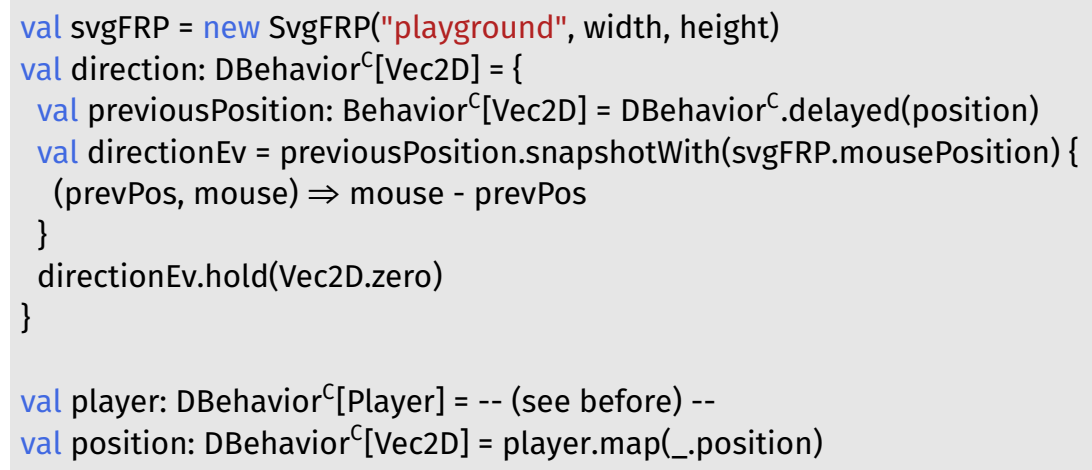

The player always moves towards the user's mouse position. We define previousPosition by delaying the player's position with DBehavior ${ }^{\complement}$.delayed, obtaining the player's position just before the current. The current mouse position is retrieved through the SvgFRP object, which represents an SVG tag in the HTML interface and makes screen-to-SVG coordinate conversions. We define directionEv as the difference between the mouse position and the previous position. The final direction behavior is then defined, taking the initial direction as Vec2D.zero.

Whether a player is attacking is simpler: we simply look if the spacebar is down using the keyboard interface.

val $\mathrm{kb}=$ new Keyboard()

val attacking: DBehavior ${ }^{\mathrm{C}}$ [Boolean] = kb.isKeyDown(" ")

The Game Interface A multi-tier FRP application is ultimately created by defining a value ui of type DBehavior' [HTML]. This behavior defines the value of the main HTML tag at every moment, and these values are rendered on screen. In this way, the programmer declaratively defines the application as "everything that is visible to the user". This main value is also a discrete behavior, i.e. it contains a notion of initial value and changes values at discrete times. Simply from this definition, the framework has all the information it needs to efficiently update the client's view.

In our example we want the interface to display the game. We obtain the SVG tag from the SvgFRP object by providing a camera and SVG tags. This camera is defined to show a fixed-size view of the game centered around the player. The behavior of SVG tags currently only contains the player's SVG representation. The resulting SVG tag is wrapped up in some HTML to produce our ui.

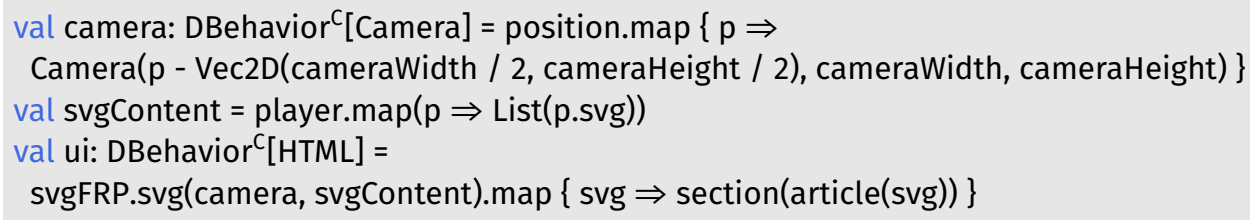

Note that we use an HTML library that exposes HTML (and SVG) tags as regular Scala functions so that - as is common in multi-tier languages - interfaces are written with the full power of a general purpose programming language. 
Listing 3 Primitives for crossing from the Client to the Session tier and vice versa object DBehavior ${ }^{\mathrm{C}}\left\{\right.$ def toSession[A](db: DBehavior $\left.{ }^{\mathrm{C}}[\mathrm{A}]\right)$ : DBehavior $\left.{ }^{\mathrm{S}}[\mathrm{A}]\right\}$ object DBehavior ${ }^{\mathrm{S}}\left\{\right.$ def toClient[A](db: DBehavior $\left.{ }^{\mathrm{S}}[\mathrm{A}]\right):$ DBehavior $\left.^{\mathrm{C}}[\mathrm{A}]\right\}$

\subsection{Multiplayer CircleRoyale}

In a multiplayer version of CircleRoyale, we do not want to compute the state of the world locally on the client. Implementing this change requires surprisingly few changes. In Gavial, going from client to server is easy with tier-crossing primitives .toSession and .toclient as shown in listing 3.

The session tier is the server-side counterpart of the client tier we've been using so far: its behaviors and events live on the server side and there is one instance of it for each client. Crossing from the client to the session tier simply sends a client's value to that client's instance of the session tier, or vice versa. Using these primitives, we change the code as follows (unmodified code in yellow).

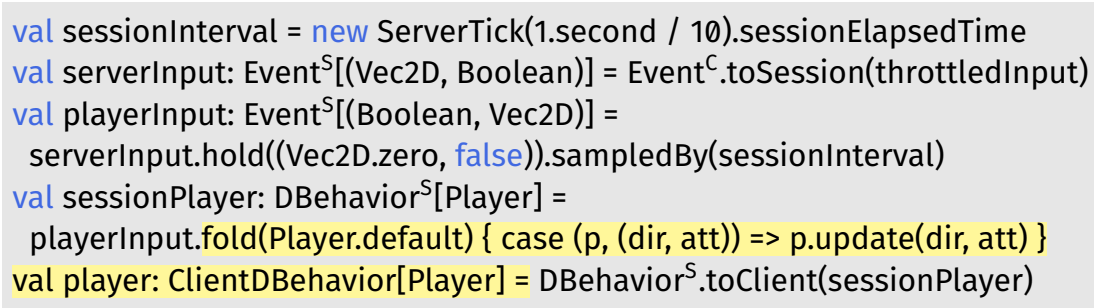

In this listing we do two things at once, (I) we do the previous computation (unmodified in yellow) at the server side on the session tier and (2) we make sure that the server computes all currently connected players (all instances of the session tier) at a rate of Io Hz. The server updates at Io Hz, even if 20 clients connect (which would produce input at $200 \mathrm{~Hz}$ ).

To do achieve these two goals, we send player input to the server using tosession and re-throttle it to Io $\mathrm{Hz}$ as before, using a server abstraction ServerTick. This rate drives the server computation and steps the game forward, sessionElapsedTime is the same across all sessions, as such everyone's game steps forwards at the same time. With server-side player input we define a sessionPlayer, with the same logic as before: user input updates the player's position and whether or not he is attacking. The client-side player definition that we used to define the interface is replaced by simply bringing the sessionPlayer to the client tier. These changes are all that is needed to construct a server-side version.

Tiers: Client, Session and Application However, CircleRoyale is still not multiplayer. To add that functionality, we need a way to combine data from different clients. Gavial offers a third tier where this is possible: the application tier. The conversion functions to the application tier expose the session tier's multiplicity: a value in the session tier corresponds to a map of values, indexed by Client as shown in listing 4. Client values act as connection tokens: they identify a browser connection to the server. In 


\section{Gavial: Programming the web with multi-tier FRP}

Listing 4 Primitives for crossing from the Session to the Application tier and vice versa object DBehavior ${ }^{\mathrm{S}}\left\{\right.$ def toApp[A](db: DBehavior $\left.{ }^{\mathrm{S}}[\mathrm{A}]\right)$ : DBehavior ${ }^{\mathrm{A}}[$ Map[Client, A]] def client: DBehavior ${ }^{S}[$ Client] $\}$

object DBehavior ${ }^{\mathrm{A}}\left\{\right.$ def toSession[A](db: DBehavior $\left.{ }^{\mathrm{A}}[\mathrm{A}]\right):$ DBehavior $\left.^{\mathrm{S}}[\mathrm{A}]\right\}$

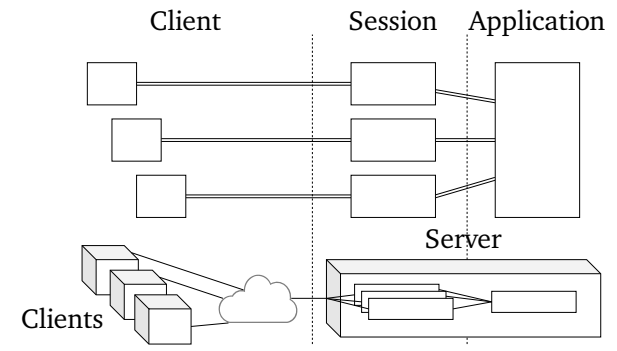

Figure 3 The tiers available in Gavial.

the session tier, the Client identifier for the current connection is available through the client primitive.

A summary of the three tiers in Gavial is shown in figure 3.

Application Tier and Client Tokens With the application tier, we can add user interaction to our game. We assume an implementation of a pure function deadClients, which performs a form of collision detection. It checks among all players that are still alive if one was hit by the weapon of another. We omit the implementation because it is not relevant to our discussion. The method returns all clients that have died (including those within the given players that were already dead).



Our previous definition of a player is no longer enough: we must now also track whether a player has died. We delay the (soon-to-be-defined) dead behavior and snapshot it with the playerinput from before and fold the result to compute a player including its .dead property. We then convert our checkedPlayer session value to an application value using toApp. The resulting behavior contains a map of all connected clients and their respective player states. The collision detection function deadClients is mapped over checkedPlayers to collect those who have lost the game, both old losers and new. With the set of dead clients, we define dead by checking whether or not the session tier's client (available through DBehavior ${ }^{\mathrm{s}}$.client) is among the dead players. 
Drawing Active Players At this point, the game is just missing an interface that shows all living players:



First, we filter out the survivors in checkedPlayers and send them to the session, and subsequently the client tier. The result is then turned into a discrete behavior of HTML tags and passed to SvgFRP.svg. Additionally, we send the existing dead to the client and use it to show the message "You died!" to dead players.

Adding a Chat Gavial also supports "regular" HTML applications that are not so heavily SVG based. To demonstrate this, we extend CircleRoyale with a minimal chat, positioned underneath the game. Users can type in their name and message and all submissions are shown in a simple list. As soon as a user submits a message, his character is labeled so that people can identify messages' authors.

A chat message is represented as a Message, a Scala case class containing a name and message and a method for converting to a string representation.

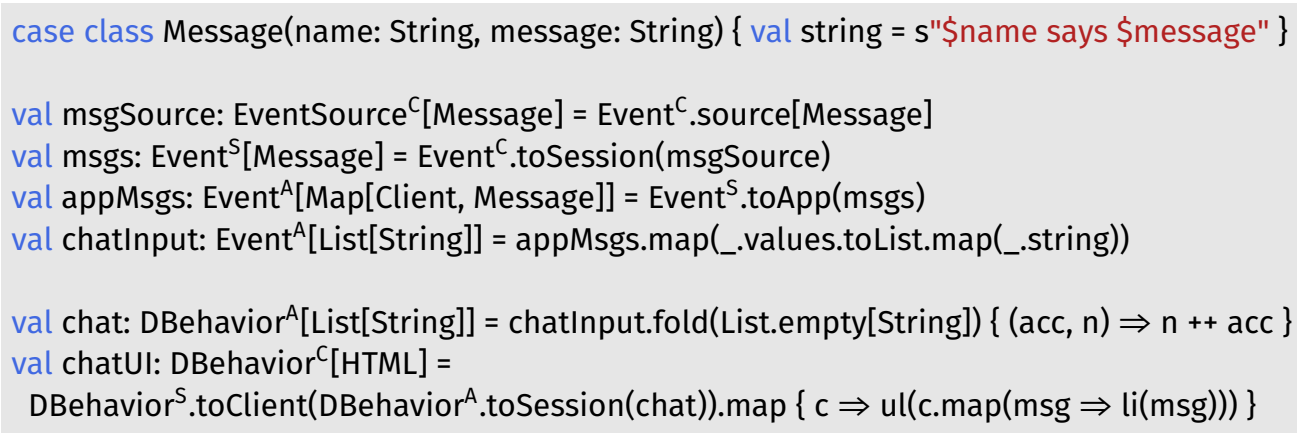

An event source is created, onto which messages can be pushed. Naturally, the chat is accumulated at the server side, by retrieving messages from the client and folding them into a list (most recent messages at the top). The accumulated chat is sent back to the client and rendered into an HTML list.

Incremental Behaviors Unfortunately, the current chat implementation has a problem: how chat is sent to the client. This discrete behavior encodes when the list changes but not how. In other words, every time a new message is added to the chat, the chat $\log$ is seen as a completely new list of strings. When we send this behavior, this means that the full list will be transmitted to the client on every update, and network traffic will grow over time.

Behaviors are a more natural encoding of the chat log (as well as having other benefits such as automatic bootstrapping of clients, see section 3.5). So to solve this without using an encoding of the chat log through events, we add an additional FRP primitive: incremental behaviors [24]. They are behaviors that not only encode when 


\section{Gavial: Programming the web with multi-tier FRP}

Listing 5 Incremental Behavior

trait Event $[A]\{$ def foldI(f: $(B, A) \Rightarrow B)$ : IBehavior $[B, A]\}$

trait IBehavior[A, DA] \{def toDBehavior: DBehavior[A] \}

object IBehavior $^{C}\left\{\right.$ def toSession $[A, D A]\left(c b\right.$ : IBehavior $\left.^{C}[A, D A]\right):$ IBehavior $\left.^{S}[A, D A]\right\}$

\section{Listing 6 DOM}

object UI \{

def listen[R](a: Attr, src: EventSource $\left.{ }^{C}[R]\right)\left(f:\right.$ js.Dynamic $\Rightarrow R$ ): AttrPair[EventSource $\left.\left.{ }^{C}[R]\right]\right\}$

object Event ${ }^{C}\left\{\right.$ def source[A]: EventSource $\left.{ }^{C}[A]\right\}$

a value changes but also why it changes, they can be used to model incremental computations and can serve as a base for other work such as incremental collections [I4]. We can also use them in Gavial to reduce the network payload.

As shown in listing 5, an incremental fold (foldl) on an event creates an incremental behavior. Incremental behaviors can be sent across all tiers, just like other FRP primitives. While they have their own (incremental) operations, for this example it suffices to know that they can be turned into discrete behaviors. We can now replace our suboptimal chat log implementation with a more efficient version, with minimal changes (marked in green, note the appearance of I s):

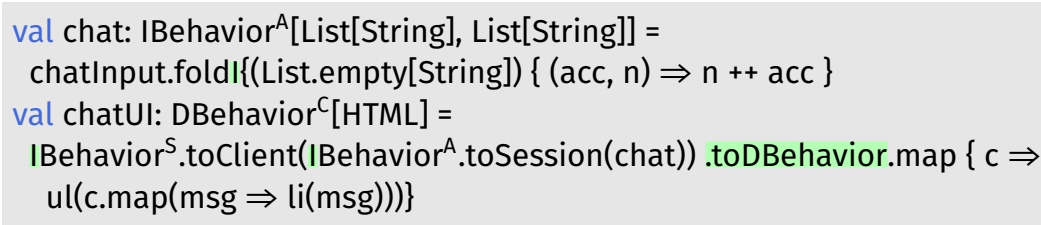

Hooking into the DOM All that is left to complete the chat is the interface. This allows us to introduce Gavial's interface to HTML elements and their event handlers.

Listing 6 shows part of the API for DOM events. Event sources are events with an "open end" and with an imperative API through which non-FRP code can inject values. UI.listen takes an extra function which turns a dynamic Scala.js value into a value of type $\mathrm{R}$ and produces an attribute pair that can be used to install the appropriate event handler on an HTML tag, e.g., button(width := "5", UI.listen(onclick, src)(_ $\Rightarrow 1)$ ).

We redefine ui a final time. The main value of our application will now show both the game interface and the chat interface. Additionally, it contains a form with a submit button that is hooked up to the msgSource event source.

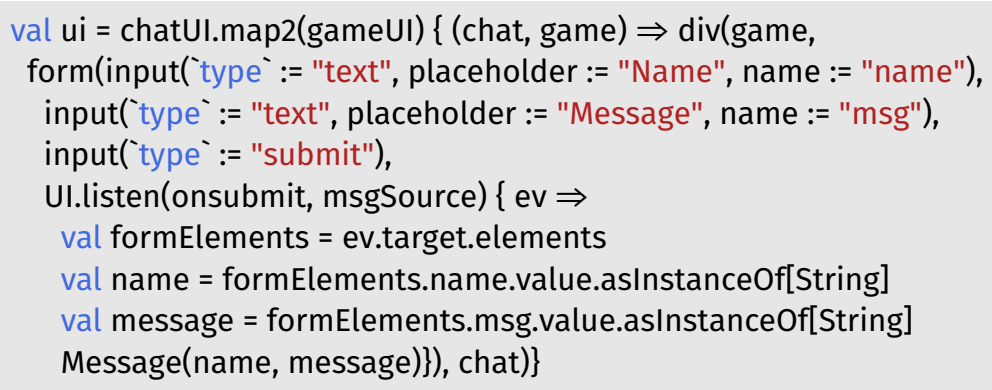


Player Labels Finally, we add player labels in game after they have posted to the chat. Although this code does not introduce new functionality of Gavial, it will allow us to explain an important aspect of tier-crossing (see section 3.6).

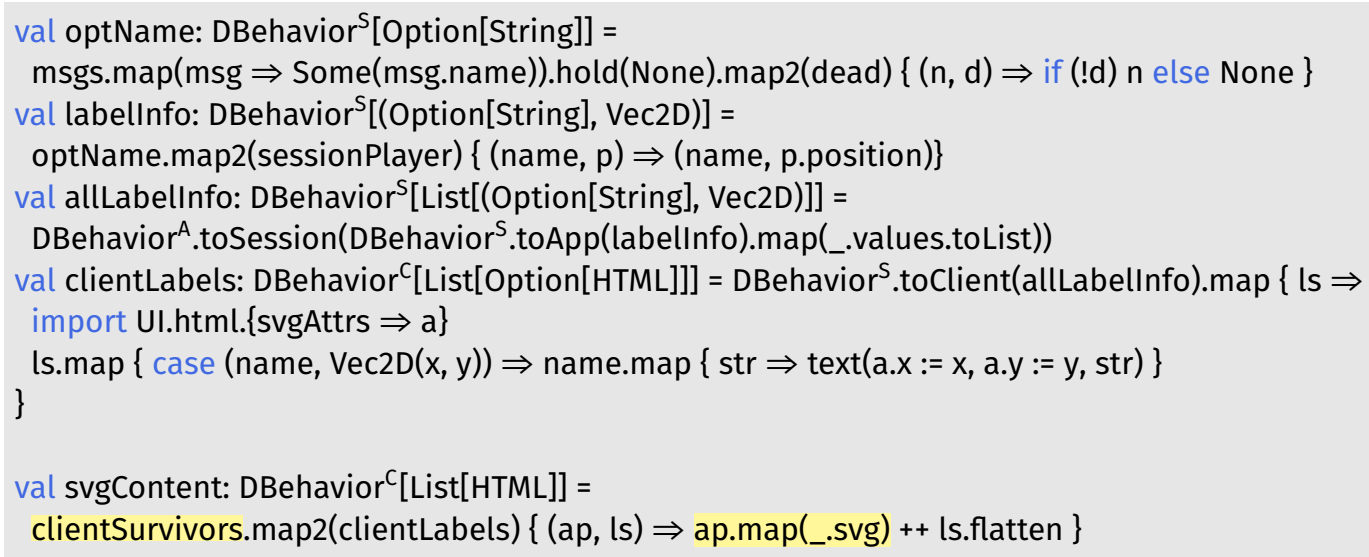

This code collects the name and position on the session tier (for living players who have already posted a message), sends it to the application tier and back (to collect all labels), and next to the client, where the non-empty labels are added to the SVG element.

\subsection{XHR or Websocket Backend}

For implementing the client-server crossing primitives, Gavial can work in one of two ways: using XMLHttpRequests or using WebSockets. The former is more widely supported and does not require a long-running open connection for each client on the server, but the latter allows bidirectional communication.

CircleRoyale does in fact use bidirectional communication. Consider, for example, the definition of svgContent in the final multiplayer example. Remember that survivors is a discrete application-tier behavior of all living players in the game. It updates at a fixed rate of $\mathrm{Io} \mathrm{Hz}$ and its new value is then pushed to all clients. Because this rate is server-initiated, our implementation requires websockets.

Nevertheless, if we want to avoid web sockets, we can modify the game so that servers do not push values to clients, but clients pull from servers. We already have the client event time, a Io $\mathrm{Hz}$ timer event. In polledPlayers, we re-use this time, send it to the server, turn it into a discrete behavior and use that behavior to read out values of survivors as polledPlayers. The polled players in turn get sent back to the client and define the new $x h$-compatible clientSurvivors.



Note again that no other code needs to change. If we make similar changes for other server-initiated session behaviors that are sent to the client, our application becomes xhr compatible. In fact, since both client-to-server and server-to-client updates are 


\section{Gavial: Programming the web with multi-tier FRP}

both driven by the time event, messages to the server and responses to the client can be exchanged in a single HTTP request.

Programmers can force xhr-mode on expressions by placing asserts which will reliably get detected during development. We discuss both backends and their requirements of each in detail in section 3.7.

\section{Making a Realistic Multi-tier FRP for the Web}

After this hands-on introduction to Gavial we take a more detailed look at its main features. This includes both existing and novel ideas and shows that FRP applied to a multi-tier web setting can benefit the development of web applications.

\subsection{Practical FRP and Incremental State}

Since there exist quite a variety of FRP flavors in academic literature and in practical implementations, it is useful to take a moment to discuss where our API can be situated in the FRP family tree and which changes were made and why, to make it usable in practice.

We support both discrete and non-discrete behaviors. The latter are behaviors that may change continuously over time or at unknown times and we do not offer, for example, a method Behavior[A].changes for them. They are evaluated as needed, similar to [I3]. This choice allows us to support behaviors that are not native to the FRP system for which changes are impossible or expensive to track, such as databases or DOM properties.

On the other hand, discrete behaviors additionally expose when a behavior changes value. An example where this is useful is the discrete client behavior ui of HTML tags. Because this is a discrete behavior, the programmer can define when the DOM should be updated. Discrete behaviors can be converted to general behaviors, simply by throwing away the "when" information.

Finally, as explained before, we also make use of incremental behaviors, which reify the fold operation on events [24] and expose not only when (like discrete behaviors) but also how a behavior changes its value. As shown in the CircleRoyale example, incremental behaviors allow us to implement efficient tier-crossing primitives without forcing programmers to use an unnatural representation of behaviors. They are created by folding events and expose both changes and deltas, the change to the behavior as a result from $\mathrm{f}$ on the event from which it was folded, and the value that initiated this change deltas.

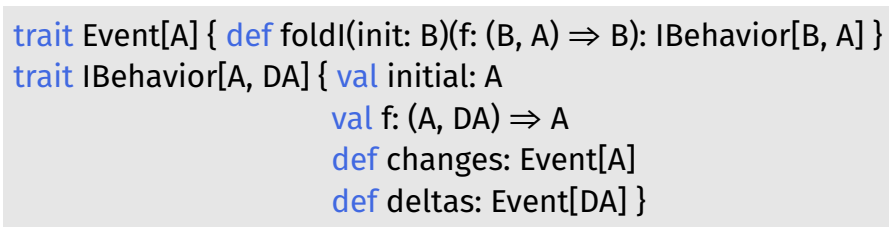

They can be converted to discrete behaviors by dropping the why information of deltas. However, discrete behaviors can also be treated as a special case of incremental 
behaviors. In this case, the changes match the deltas and the folding function $f$ simply ignores the older value while using the new value: $\left(_{-}, a\right) \Rightarrow a$. We use this property frequently to re-use incremental behavior specific APIs.

First-order FRP We also limit our FRP to first-order FRP (as opposed to higher-order FRP). In other words, we do not offer APIs like flatten: Behavior[Behavior[A]] $\Rightarrow$ Behavior[A] that flatten nested FRP abstractions. First-order FRP is conceptually simpler, because dependencies between behaviors and events are statically known. These guarantees make it suitable for multi-tier FRP, as dynamically generated client/server crossing would be hard to understand and implement. It also avoids certain tricky problems of higher-order FRP, like the so-called time leaks that cause memory leaks in naive higher-order APIs (see, for example [32]), and we do not need to modify our API to prevent them. On the downside, first-order FRP is less expressive, but as shown in [36], the full expressiveness of higher order FRP is not always necessary.

\subsection{Tiers}

Our API is tailored to the standard web distribution model, where there is essentially one server and an arbitrary number of active clients (browsers) that connect to the server. We assume that these clients are only active for a subset of the application's lifetime and we distinguish the programs in multiple tiers. Previous work in multi-tier languages in general [8, I9, 30, 6, I] or specifically in multi-tier FRP [25] work with two tiers: client and server. A problem with a two-tiered system is that the framework makes a decision to focus itself to one style of programs. Regular request-response interaction between the client and the server is easier if the chosen server tier is most akin to the session tier. Typical create-read-update-delete applications fall in this category. On the other hand, applications that rely heavily on user-to-user interaction through the server are more difficult to write and have to imperatively manage state across clients somehow. Such interactive applications are easier to write if the server tier is most akin to the application tier since sharing state across clients is part of the programming model. However, programs that primarily focus on handling a single client's requests become very tedious to write.

As such, we support both types of programs in an equally convenient way through the three tiers previously explained and illustrated in figure 3: the application (single instance, server-side), session (client-specific, server-side) and the client tier (clientspecific, client-side). The server keeps track of every active client connection and assigns it a unique identifier. This value is exposed in the API as opaque values of type Client and shows up in certain tier-crossing primitives (e.g., .toApp) as well as in the following primitives:



The client primitive exposes a session's Client as a session behavior. The application event clientChanges informs about clients connecting or disconnecting. We use Scala's 


\section{Gavial: Programming the web with multi-tier FRP}

sealed traits to encode the event information (the Client and whether it just connected or disconnected):

sealed trait ClientChange $\{$ val client: Client $\}$

case class Connected(client: Client) extends ClientChange

case class Disconnected(client: Client) extends ClientChange

\subsection{Crossing Application \& Session Tier}

When sending events or behaviors between the session and application tier, the primitives need to deal with the fact that the session tier exists in many copies at the same time (one for each active client), while there is only one instance of the application tier.

This is reflected in the type of the session/application tier-crossing primitives for events:

object Event ${ }^{S}[A]\left\{\right.$ def toApp(e: Event $\left.{ }^{S}[A]\right):$ Event ${ }^{A}[$ Map[Client, A]] \}

object Event ${ }^{A}[A]\left\{\right.$ def toSession(e: Event $\left.{ }^{A}[A]\right):$ Event ${ }^{S}[$ Map [Client, A]] $\}$

Sending a session event to the application tier produces an ApplicationEvent for a different type of values: Map[Client,A] instead of A. Intuitively, the event .toApp(e) will fire whenever at least one of the copies of the session event e fires and a map will be produced containing the identifier of the connection and the event value for each of these copies. Conversely, an application event e can be sent to a session event of type Event ${ }^{\mathrm{S}}[\mathrm{A}]$ where it fires for each client whenever e fires.

The situation is similar for sending behaviors.

object Behavior $[A]\{$ def toApp(b: Behavior $[A]): B^{S e h a v i o r}{ }^{A}[$ Map[Client, A]] $\}$ object Behavior ${ }^{A}[A]\left\{\right.$ def toSession(b: Behavior $\left.{ }^{A}[A]\right):$ Behavior $\left.^{S}[A]\right\}$

Sending a session behavior to the application tier creates a Behavior ${ }^{\mathrm{A}}[$ Map[Client,A]] which maps active clients to their value of the behavior. The converse primitive simply produces a behavior with the same value for every client.

Incremental \& Discrete Behaviors Sending incremental behaviors between the session and application tiers is a bit more complicated. We do not discuss discrete behaviors, they can be seen as a special case of incremental behaviors and all techniques discussed here are valid for those.

Consider first a session incremental behavior $\mathrm{b}$ and think about what the type of IBehavior ${ }^{\mathrm{s}}$.toApp(b) should be if $b$ has type of values of $A$ and type of deltas DA. As before for regular behaviors, the type of values for Behaviors.toApp(b) should naturally be Map[Client,A]: a map containing for every active client the value of the corresponding copy of the session incremental behavior. But now we should choose a type of deltas that can represent any way in which the value initiates change. Obviously, one or more of the copies of $b$ may change, so we need this type to contain a Map[Client, DA]. However, another possible cause for the value to change is that clients have connected to the server, in which case a new copy of the session behavior will be made and a new entry in the map will appear, and conversely clients may disconnect in which case a 
copy will be dropped and an entry will disappear. This is why the type of deltas should be (Map[Client, DA], Option[ClientChange]), that is, each delta is a map of client specific changes (possibly empty) and a possible change in client connections. Both deltas can also appear at the same time, for example, if the value delta is derived straight from the clientChanges primitive. The complete type of the method:

object IBehavior ${ }^{\mathrm{S}}\left\{\right.$ def toApp[A, DeltaA](sb: IBehavior ${ }^{\mathrm{S}}\left[\mathrm{A}\right.$, DeltaA]): IBehavior ${ }^{\mathrm{A}}[$ Map[Client, A], (

$\hookrightarrow$ Map[Client, DeltaA], Option[ClientChange])] \}

The other way around, sending an application incremental behavior to the session tier simply sends values and deltas directly:

object IBehavior $^{A}\left\{\right.$ def toSession[A, DeltaA](sb: IBehavior ${ }^{A}\left[A\right.$, DeltaA]): IBehavior ${ }^{\complement}[A$, DeltaA] $\}$

Note that crossing from the session to the application tier is done in-memory. As such, bandwidth overhead is less of an issue and we expect the incremental session to application API to only be used in applications where incremental behaviors are used to also reduce computational overhead. If computational overhead is not a main issue, incremental behaviors can be turned into discrete behaviors with major simplifications in the API (identical to Behavior ${ }^{\mathrm{s}}$ ).

\subsection{Crossing Client \& Session Tier}

Being able to send events and behaviors from a client tier to a server tier is one of the key features of our model. We do not offer tier-crossing primitives for non-discrete behaviors between the client and session tier. The reason is that we want to use only asynchronous communication between client and server. Imagine a tier-crossing primitive for a client (non-discrete) behavior $b$ to the server side as b.tosession. It is generally impossible to predict upfront at the client side when the value of b.toSession will be required on the server, so the only possible implementation would have the server synchronously request the current value to the client and block execution until the client answers.

The client/session tier-crossing primitives need to transmit values across the network. This requirement shows up in the API as type-classes encoded as Scala's implicit arguments [2I, 20]. All values that cross the network are required to be serializable, visible in our API as extra requirements on the type in form of: A: Encoder: Decoder. We use an existing Scala library to supply encoders and decoders for standard items and for case classes (semi-)automatic derivation is available.

Between the client and session tier, sending events is straightforward. A client event e of type Event ${ }^{\mathrm{C}}[\mathrm{A}]$ can be sent to the server as .toSession(e) of type Event ${ }^{\mathrm{S}}[\mathrm{A}]$ and vice versa using .toclient(e). Intuitively, when the event e fires, it asynchronously sends to the other end of the tier boundary. At that other end, the event fires from the sent event after the network delay when its received.

object Event ${ }^{\mathrm{C}}\left\{\right.$ def toSession[A: Decoder: Encoder](e: Event $\left.{ }^{\mathrm{C}}[\mathrm{A}]\right)$ : Event $\left.{ }^{\mathrm{S}}[\mathrm{A}]\right\}$ object Event ${ }^{\mathrm{S}}\left\{\right.$ def toClient[A: Decoder: Encoder](e: Event $\left.{ }^{\mathrm{S}}[\mathrm{A}]\right):$ Event $\left.^{\mathrm{C}}[\mathrm{A}]\right\}$

For an incremental behavior $\mathrm{b}$, it is known when the value changes, so we can produce correct values at the other side of the network for the sent behavior .toSession(b) or 


\section{Gavial: Programming the web with multi-tier FRP}

.toclient(b) simply by sending an update whenever b changes. As explained before, this does not require transmitting the full value of behavior $b$, but just the deltas that represent what has changed. We can re-compute the new value on the server from the previous value and the delta. In other words, if $b$ has type IBehavior ${ }^{C}[A, D A]$, we only need to transmit the value of type DA when $b$ changes and the result is an incremental behavior of type IBehavior ${ }^{\mathrm{S}}[\mathrm{A}, \mathrm{DA}]$. Of course, when the client first connects, there is no point in transmitting a delta and we transmit the full initial value.

object IBehavior $^{\mathrm{S}}\left\{\right.$ def toClient[A: Decoder: Encoder, DeltaA: Decoder: Encoder](b: IBehavior ${ }^{\mathrm{S}}[\mathrm{A}$,

$\hookrightarrow$ DeltaA]): IBehavior ${ }^{\mathrm{C}}[\mathrm{A}$, DeltaA] $\}$

object IBehavior ${ }^{C}\left\{\right.$ def toSession[A: Decoder: Encoder, DeltaA: Decoder: Encoder](b: IBehavior ${ }^{\mathrm{C}}[\mathrm{A}$, $\hookrightarrow$ DeltaA]): IBehavior $[$ A, DeltaA $]\}$

\subsection{Bootstrapping Clients}

One of the useful properties of combining FRP with multi-tier languages is automatic bootstrapping of clients [25]. Bootstrapping is the initial provisioning of client values with the latest state of session behaviors sent to the client. It is a standard task in web application development, typically solved in an application-specific way by for example, embedding initial values in the HTML or by polling for the latest values at client startup. The multi-tier FRP abstractions of Gavial allow us to solve the bootstrapping in a general, natural and transparent way.

This property is a direct result of the (natural) semantics of .toSession on incremental behaviors, which define the initial value of an incremental session application behavior $\mathrm{b}$ as the value of $\mathrm{b}$ at the connection time of the client. If an application behavior sent to the session tier is further sent to the client, the client will also be provisioned with this value. This saves developers the work of implementing manual initialization schemes and automatically helps them define the initial state of new clients.

\subsection{Tiered Glitch Freedom with Minimal Overhead}

Something that we have not discussed before are the guarantees of the tier-crossing primitives and how they differ from regular FRP semantics. Correctly implemented FRP libraries follow FRP semantics and protect programmers from partial event propagation. For example, you would expect $t$ in the following expression to remain true throughout updates to $\mathrm{x}$ :

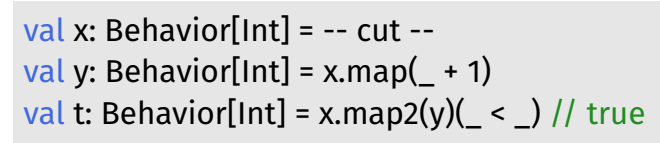

Propagating $\mathrm{x}=20$ from $\mathrm{x}=1$ should evaluate $20<21$ for $\mathrm{b}$ instead of ever ending up in $20<2$ or $1<21$. If such partial updates can be observed, they are called glitches. Our proposed multi-tier FRP has a similar property for network-crossing primitives. To explain this, we visualize propagation of the above toy example as a graph in figure 4 and add a network between $x$ and $y$, and $t$. This corresponds to the code in the left part of figure 4 

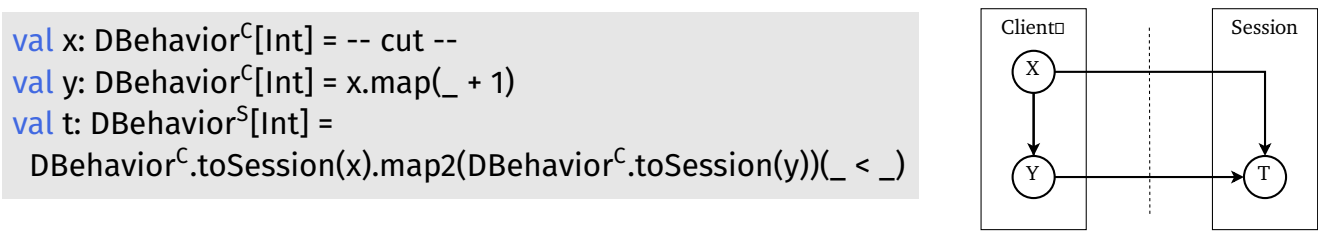

Figure $4 \mathrm{x}<\mathrm{y}$ across tiers

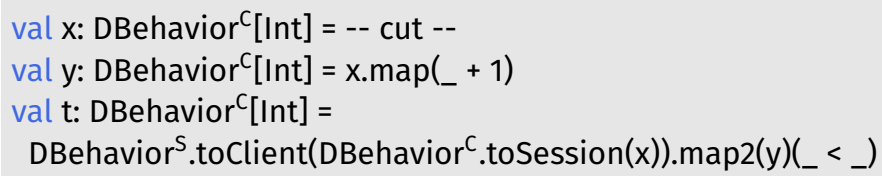

Figure $5 \mathrm{x} \nless \mathrm{y}$ across tiers

In a naive implementation of multi-tier FRP (such as $[25,23,4]$ ) glitches would inevitably occur in t due to network delays, however in our proposal neither client to server communication nor server to client communication results in glitches? In Gavial, all events or behaviors that cross from the client to the session tier and vice versa are propagated atomically. This means that $\mathrm{t}$ will always be true, no partial updates can ever reach the session tier.

Glitches are still possible, but only if FRP values depend on values of different tiers, for example as follows:

In this case, there will be a delay between the updates of $y$ and the version of $x$ sent to the server and back. We purposefully do nothing to hide such network delays, as that would require the computation on the client (or server if the situation was reversed) to be blocked until the full propagation catches up). We leave it up to the programmer to take network delays into account. In other words, we prevent those forms of glitches that can be prevented without working around the distribution model of the web. We will refer to this property as "Tiered Glitch Freedom". It is not the purpose of this paper to express or prove this property formally, but it is in fact expressed by our denotational semantics (see section 3.9).

Perhaps surprisingly, we rely on this property in two cases of the CircleRoyale example. Once in the interaction of defining direction and showing all players on the user interface and another time in the interaction between naming players and showing them. In both of these cases we are sure that: (I) a player's direction is calculated based on the position that is actually shown in the playing field and (2) a name in the chat is always drawn at the same time on the playing field. There can be no consistency mismatches between names, direction or what is visible in the playing field even though we conceptually do three separate tier-crossings: sessionPlayer (for direction), clientSurvivors (for drawing players) and name (for labeling drawings). Our tier-crossing primitives guarantee that all values cross the network atomically within

\footnotetext{
${ }^{3}$ With the exception that it is possible to make use of the FFI or Async to intentionally separate
} network propagation for efficiency. 


\section{Gavial: Programming the web with multi-tier FRP}

one propagation cycle. This property allows programmers to more safely refactor and add to existing multi-tier FRP code with the added guarantee that data does not get propagated in unexpected ways. With a naive multi-tier FRP implementation we would have had to go back and manually batch updates into a single toclient call. This same property is available in the other direction as well, all tosession crossings are done in the same atomic manner.

Tiered glitch freedom gives us strong guarantees: manipulating behaviors or events on one tier and then sending to another is equivalent to first sending and then manipulating them. For example, the previous definition of $\mathrm{t}$ in figure 4 is (functionally) identical to the following definition:

val t: DBehavior ${ }^{\mathrm{S}}[$ Int $]=$ DBehavior $^{\mathrm{C}} \cdot$ toSession$\left(\mathrm{x} \cdot \operatorname{map} 2(\mathrm{y})\left({ }_{\_}<\_\right)\right) / /$true

Our approach avoids a large implementation cost (as seen in section 4) but still provides useful guarantees. It forms a middle ground between two extremes "local glitch freedom" and "total glitch freedom" that we call tiered glitch freedom.

Local Glitch Freedom Client/server web applications are usually treated as separate programs and previous work on multi-tier FRP also treats client and server programs essentially as distinct but connected FRP applications [25, 23, 4]. Instead of having consistency guarantees regarding glitches, cross-tier connections are treated as a communication channel that lies beyond the scope of the FRP semantics. In practice this means that events are transmitted as soon as they come in but the guarantees we have for $t$ in figure 4 do not hold.

Total Glitch Freedom The other extreme alternative to our tier-crossing semantics is glitch-freedom in a distributed setting [II, I6, I8]. In other words, across the distributed reactive program there can never be an observable partial propagation. To make this possible there are extra restraints on the propagation, certain projects require a global lock on the whole program, others block and queue propagation in certain subgraphs. In a web settings, for example if input validation is done on the server, then these semantics require that input from the client is transmitted to the server, validated and transmitted back, all in the same propagation, so that the client and server both block until everything is finished. To combat these issues, projects such as DREAM [16] and ScalaLoci [35] make these algorithms pluggable, but none have tiered glitch freedom as an option.

\subsection{XHR or WebSockets?}

A novel aspect of Gavial is that it automatically selects the network communication backend to use based on the primitives used to write the program. The xhr-mode can be used as long as the application does not require the server to initiate communication with a client. WebSocket mode becomes a requirement as soon as functionality cannot be implemented in a request-response style manner. Intuitively this happens in two cases: (I) whenever a server sends something to a client on its own (through timers 
Listing 7 Imperative FRP API



or through the foreign function interface) or (2) whenever clients send information to other clients through the server.

To decide whether such cases are present, Gavial analyses the FRP graph and tags every event or behavior as "needing bidirectional communication" or not. Operations such as map simply take the mode of the parent event or behavior. Operations that combine multiple events or behaviors such as map2 take the most restrictive mode of its dependencies, if one requires bidirectional communication then so does the result.

An exception to this rule are the snapshotwith operations. Snapshotting a behavior $\mathrm{b}$ that requires bidirectional communication with an event e that does not, produces a result that does not require bidirectional communication either. This makes sense because changes in $b$ will not cause changes in b.snapshotWith(e).

Calculating whether or not the bidirectional communication is necessary is done at startup time and developers can place asserts to force xhr-mode. The same functionality can be modeled in the Scala type-system, however, this would occur everywhere in the API and would clutter it significantly. We opted for a run-time implementation that does not use the type-system, however xhr-mode violations are reliably detected during development and stops execution with the appropriate error message before the web-server becomes available.

\subsection{Interacting with the World}

We have seen a glimpse of how to interact with the world in section 2. However, most of the actual interaction was hidden behind some convenient abstractions such as SvgFRP. While these foreign APIs are not part of the core design effort, they make Gavial realistic and practical.

Connecting to non-FRP APIs There are three main ways of interacting with non-FRP APIs from within the FRP system, through event sources, by polling behaviors and through behavior sinks as shown in listing 7 .

Event sources are "open" events. They have the added ability of being triggered imperatively through an "engine". The engine is an exposed value of the underlying FRP library and contains a fire method that starts a propagation cycle in the FRP network. Another way of making an event source is through the sourceWithEngineEffect method, this requires a function that gets a function as a parameter of type $A \Rightarrow$ Unit. The given function imperatively fires a value onto the event source that is created through the method and allows programmers to conveniently write code that interacts with the DOM, for example an excerpt of the Keyboard class: 
developers to communicate asynchronously with external services such as other web APIs or databases without blocking the FRP program.

\subsection{Denotational Semantics}

While we hope that the API is intuitive and easy to comprehend, it is of course important to specify the semantics of the API completely and precisely. We defined a denotational semantics for Gavial as a non-ambiguous reference specification of the core APIs. Time and network delays were modeled and the semantics were actively used during API design. They helped us get the types of the tier-crossing APIs right and gave us useful insight when dealing with corner cases (particularly related to bootstrapping, see section 3.5). The denotational semantics do not play a large part in this paper and we do not use them to prove novel properties, but they were helpful as an implementation specification and might be helpful as a reference to the reader, it is available as supplemental material on https://doi.org/10.5281/zenodo.3647731.

\section{Implementation}

\subsection{Embedded as a Library in Scala}

Gavial is completely embedded in Scala in order to use existing libraries and it makes use of Scala.js [Io], a Scala to JavaScript compiler. It is set up using the same techniques used in the Scalagna project [27], an experimental multi-tier-as-a-library for Scala. Gavial is implemented as two Scala libraries: a JVM and JavaScript library, as well as some common shared code. The server and client-side FRP primitives are respectively backed by real and mock implementations on the server and vice versa on the client and we make sure tier-crossing primitives are supported appropriately on each side. For more detail on how we use both compilers we refer to appendix A.

From a Gavial user's perspective, a program is a cross-build between two environments - the JVM and JavaScript backends - which is supported in the Scala build tool (SBT) using plugins. Since Gavial is just a library this means that a developer gets nice integration into known production quality Scala tools in comparison to creating a new multi-tier language from scratch.

Reusing the Scala/Scala.js Ecosystem Developers can also make use of the entire Scala/Scala.js ecosystem of libraries as well as the JVM and JavaScript ecosystems through the corresponding Scala FFIs. Libraries that are only supported for one backend can be integrated, either in a backend-specific source file, or by using special (and somewhat crude) annotations @client and @server. @client-annotated code is not compiled on the server and vice versa. 


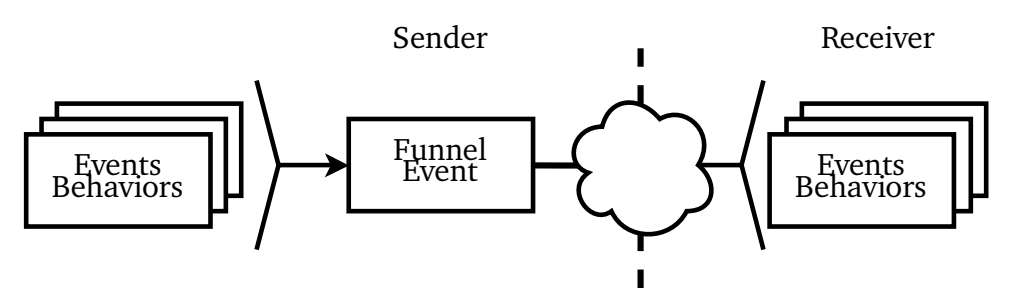

Figure 6 Efficient Tiered Glitch Freedom

\subsection{Efficient Tiered Glitch Freedom}

As we have seen, client-server communication should behave with minimal glitches. To achieve this, all sent behaviors and events have a unique identifier, which is the same in the client and server code. To cross tiers with tiered glitch freedom we merge all events and behaviors into one large funnel event on the sender side. On the server-side, it is of type Client $\Rightarrow$ List[Message] and contains a function that for all clients produces an (optionally empty) list of messages. Messages consist of the tier-crossed event or behavior's identifier and a value. At the receiver's side, a message router receives this list of messages, splits it up into updates that can be fed into the local FRP engine in a single propagation cycle. This whole process is shown in figure 6 . This shows that implementing tiered glitch freedom is low-cost both in implementation as well as in performance cost in an FRP program.

Backends The exact implementation depends on the backend the program is running on. The websocket backend simply uses bidirectional communication as expected. Whenever a propagation cycle ends on one end the changes are sent to the other and vice versa. The xhr backend works a bit different since client propagation cycles take care of both directions of communication. At any time the client propagation cycle requires events to be propagated, a request is sent to the server. The server running in xhr-mode then executes a propagation cycle which creates new values that should be shipped back to the client.

Performance A small test in our student lab on CircleRoyale (the full version on websockets available on github.com/tzbob/circleroyale) was run on one PC while continuously adding players to the game. On an unoptimised single-threaded version of CircleRoyale, we were able to sustain 35 concurrent clients, i.e. $\approx 350$ client-toserver messages and $\approx 2$ IOO server-to-client messages per second. The most taxing operations were in collision detection (naive implementation) and the underlying messaging library. This limited test indicates that Gavial does not impose a large overhead compared to the underlying tried-and-tested Scala libraries.

\subsection{Crossing Tiers with Incremental Behaviors}

Propagating changes of behaviors is entirely similar to those of events. However, extra support is needed to replicate initial values. Similarly to merging all changes into one big event, behaviors are all bundled into a single behavior of type Client $\Rightarrow$ List[Message]. 
Table 1 Comparison Table for Multi-tier Reactivity: $\checkmark \rightarrow$ has feature; $X \rightarrow$ does not have feature; empty $\rightarrow$ not applicable; other $\rightarrow$ in table

\begin{tabular}{|c|c|c|c|c|c|c|c|}
\hline Project & $R e^{a c t i v e}$ & ${ }_{R P} P^{T} \mathrm{Pe}^{\mathrm{x}}$ & consists & orethe & [ncre & Web API & $\mathrm{BaSe}^{\mathrm{LanOadad}}$ \\
\hline Gavial & Both & $\checkmark$ & Tiered & $\checkmark$ & $\checkmark$ & $\checkmark$ & Scala \\
\hline $\mathrm{Ur} / \mathrm{Web}$ & Client & To Client & Tiered & $\checkmark$ & $\mathrm{x}$ & $\checkmark$ & From scratch \\
\hline ScalaLoci & Both & $\checkmark$ & Flexible & Flexible & $\mathrm{x}$ & & Scala \\
\hline DREAM & Both & $\checkmark$ & Flexible & Flexible & $\mathrm{x}$ & & \\
\hline QPROPd & Both & $\checkmark$ & Total & $\mathrm{x}$ & $\mathrm{x}$ & & \\
\hline SID-UP & Both & $\checkmark$ & Total & $\mathrm{x}$ & $x$ & & \\
\hline Eliom & Both & $\checkmark$ & Local & $\checkmark$ & $x$ & $\checkmark$ & OCaml \\
\hline Scala Multi-tier FRP & Both & $\checkmark$ & Local & $\checkmark$ & $\mathrm{x}$ & $\checkmark$ & Scala \\
\hline AmbientTalk/R & Both & $\checkmark$ & Local & $\checkmark$ & $\mathrm{x}$ & & From scratch \\
\hline Flask & Both & $\checkmark$ & Local & $\checkmark$ & $x$ & & Haskell \\
\hline (Нip)Нор & Both & $\checkmark$ & & & & $\checkmark$ & Scheme \\
\hline (Hip)HopJS & Both & $\checkmark$ & & & & $\checkmark$ & JavaScript \\
\hline
\end{tabular}

The data to properly initiate the incremental behaviors is sent when a client connects to the server. Exactly when and how depends on the backend. In websocket mode the initial data is pushed from the server to the client as soon as a connection is made. In xhr-mode a request is sent from the client as soon as the client-side program is loaded.

\section{Related Work}

In this section we discuss distributed reactive and/or multi-tier programming languages and relate them to our work. We do not go into detail on multi-tier language proposals that do not have reactive programming features such as the initial proposed multi-tier calculus or later additions [19, 7] nor ML5 [34], Links [8] or automatic slicing techniques such as Stip.JS [22].

Regarding multi-tier languages, we look at languages that are based on existing languages such as Eliom [23] (OCaml), ScalaLoci [35] (Scala), Hop [29, 3I] (Scheme), HopJS [30, 33] (JavaScript) and Flask [I5] (Haskell) as well as languages that are built from scratch such as Ur/Web [6, 5] and AmbientTalk/R [4].

In the field of distributed reactive programming there are several programming languages or even algorithms that describe systems relevant to our multi-tier implementation of FRP with tiered glitch freedom such as SID-UP [II], DREAM [I6] and QPROPd [I8].

We focus on whether or not there is support for reactive programming on the client, the server, both or on flexible tiers ${ }^{4}$ (also written as both). For these flexible projects

\footnotetext{
${ }^{4}$ Some multi-tier languages support distributions other than the client/server architecture of the web.
} 


\section{Gavial: Programming the web with multi-tier FRP}

we specifically look at the availability of reactive tier-crossing primitives and the consistency properties thereof.

\subsection{Local Reactive Programming in Multi-tier Languages}

Both Hop (based on Scheme) and its successor HopJS (based on JavaScript) have reactive programming libraries named HipHop(JS) [3, 33] respectively. The HipHop libraries are based on synchronous programming languages such as Esterel by [2] and make it possible to create reactive programs in a synchronous DSL similar to Esterel. Synchronous programs are written in isolation and plug into the regular Hop execution as input to output event processors. HipHop supports both execution on client and server-side of Hop but does not provide any means to create one conceptual reactive program across tiers and thus does not provide a means for automatic bootstrapping nor any cross-tier reactive consistency guarantees.

Ur/Web provides a source that can be compared to an EventSource that we discussed throughout the dissertation. It has the same imperative functionality as references, you can create them and set or get its value. Only creation or setting the source is supported on the server. Composing sources is done by "subscribing" to a source and creating a signal. Such a signal allows composable reads over several sources and can be embedded into Ur's HTML pages. This gives developers an imperative RPC-style interface from the server to a client-side FRP program. The entire page is created from the current source values and as such Ur/Web has a similar elegant solution to the bootstrapping problem we describe in section 3.5 .

\subsection{Multi-tier Reactivity}

Embedding reactive programming in multi-tier programming by making it possible to write reactive programs in each tier is a first step. Several languages go further (like we do) and allow building a reactive program that spans all tiers with primitives to cross tiers. We divide related work in three sections of multi-tier reactivity, those that provide local glitch freedom, total glitch freedom and tiered glitch freedom.

Local Glitch Freedom The simplest form of tier-crossing primitives provide local glitch freedom.

Multi-tier FRP in Scala [25] provides .to(Client/Server) on events and discrete behaviors, but naively connect client FRP applications to server FRP applications without minimizing glitches or providing any consistency guarantees. They solve the bootstrapping problem as we do in section 3.5 .

Eliom provides a client/server reactive abstraction (since v5.0) ${ }^{5}$ They provide a client signal that can be initialized on the server and used on the client. They also provide a server signal and have similar tier-crossing primitives that naively propagate events from one tier to the other. As such, they provide a similar solution to the

\footnotetext{
${ }^{5}$ https://opam.ocaml.org/packages/eliom/eliom.5.0.0/
} 
bootstrapping problem as well as a multi-tier reactive programming environment similar to [25].

An extension of AmbientTalk/R to combine the advantages of loosely-coupled publish/subscriber systems with the elegance of reactive programming constructs is explained in Loosely-Coupled Distributed Reactive Programming in Mobile Ad Hoc Networks by [4]. They provide ambient behaviors which is a construct that allows the propagation of events to reactive values hosted on other reactive networks by means of publish/subscribe. An ambient behavior is a behavior that is subscribed to previously exported behaviors. Our approach can be compared to theirs by looking at to(Client/Server) as a combination of export/subscribe. Since we assume a 'single server with multiple clients' architecture we greatly simplify our API, as a result we do not provide the flexibility that AmbientTalk/R provides.

Flask is not a multi-tier language applied to the client/server web, it is a distributed FRP language for sensor networks. They provide support for broadcast topologies and have no consistency guarantees regarding propagation.

Total Glitch Freedom Other than specifically targeting web development, academia also focused on a more general distributed reactive programming (DRP) with the aim of providing alternatives to the Observer pattern in a distributed environment. An overview of requirements and challenges of DRP is provided in Towards Distributed Reactive Programming by [28]. The projects we compare with in this space are not multi-tier languages specifically targeted toward the client/server nature of the web. They are targeted towards a larger distribution pattern of a reactive program where, e.g., multiple distributed reactive expressions make up a single program. In our distributed multi-tier project it is about how to unify a client and a server reactive program. Nonetheless, the programming models they build and propose are very related to our multi-tier reactive programming.

A DRP approach that focuses strongly on consistency guarantees is defined in [I6]. They deliver three levels of consistency guarantees: causal, glitch free and atomic. Causal consistency refers to propagation that maintains causality within one process, e.g., $e_{1}$ happens before $e_{2}$ in the origin reactive nodes and will only be able to be observed in that order by other reactive nodes. Glitch free consistency means that a partially propagated FRP network is never observable, even in the distributed setting. Finally, atomic is a consistency guarantee that delivers total FIFO ordering and glitch freedom and thus is the most expensive of them all. Their implementation for glitch free consistency (including atomic, which adds distributed locking to it) requires cross tier propagation messages to include extra details (the history of the propagation) which causes the network traffic to increase. While their consistency guarantees are flexible, they do not provide a consistency guarantee that is similar to tiered glitch freedom.

Several other distributed reactive algorithms were proposed with similar goals. SID-UP [II], a distributed glitch-free propagation algorithm that minimizes messages compared to DREAM and requires a centralized "lock" that makes the distributed program unable to process more than one propagation at a time. QPROP [I8], an 


\section{Gavial: Programming the web with multi-tier FRP}

algorithm that provides distributed glitch-free propagation that does not require a central coordinator for locking.

ScalaLoci is not a multi-tier programming language applied to the web, however, it is very related to our work. They also target the Scala language and also do this without modifying the compiler. Instead of having two (or three) set tiers they provide a type system in which a programmer can express the distribution of the program. The placement types are used to define on which location certain expressions live and they support reactive programming with tier-crossing primitives. The consistency guarantees of these tier-crossing primitives are flexible and pluggable, so far they support SID-UP and a propagation similar to [I7] which provides no distributed guarantees. We think a version of tiered glitch freedom would complement the project well.

Tiered Glitch Freedom Ur/Web provides client-side reactive programming but also has an interesting consistency property for its server-to-client tier-crossing primitives. There are no formal semantics on the Ur/Web RPC calls but if we understand correctly, Ur/Web provides a consistency property similar to tiered glitch freedom in the direction of server-to-client. Ur/Web's programming model is tied tightly to the request-response style of the web, all server-to-client communication within a response of a client-toserver RPC call is done atomically.

In comparison, our work has the same consistency guarantee, but instead of only providing it in one direction, our tiered glitch freedom (see section 3.6) has the same guarantee in both directions.

Incremental Propagation None of the multi-tier reactive languages and algorithms we described document support for incremental propagation. They have no primitives similar to our .toclient/Session for incremental behaviors. As such, incrementally built behaviors such as an incremental collection propagate their full state instead of their change.

Three Tiers Our multi-tier reactive programming model is split into three tiers. As earlier explained in section 3.2, the motivating factor is to cope with both requestresponse style applications as well as user-to-user style applications. In ScalaLoci it is possible to express "tiers" in the program manually, for example our client-sessionapplication tiered system:

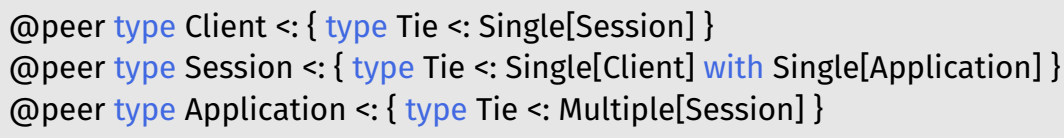

The APIs they provide have similar types as ours, that is, crossing from a single tie to a multiple tie shows the multiplicity in the primitive's type. Signal[T].asLocalFromAll on a session signal would return a Signal[Map[Remote[Session], Signal[T]]]. Glitch-freedom between the session and application tier for our project is a given since they run in the same FRP application on the server. As far as we know, ScalaLoci does not take into account deployments that are physically on the same JVM, so a session to application 
model we describe would incur the same overhead or glitches as the client to session tier connection would.

\section{Conclusion and Future Work}

In this paper, we have focused on the idea of multi-tier FRP (specifically for the web's client-server architecture) with asynchronous tier-crossing primitives. Several existing and novel ideas in both FRP and multi-tier research fit together to form Gavial. The core API and primitives (FRP with crossable tiers) were enriched with asynchronous behaviors, APIs to work with imperative programs, HTML support, etc., to support real-world applications. Novel ideas such as a three-tier model, minimal glitches and support for XHRs are available as a library to be used in the matured Scala toolchain. While the main emphasis of Gavial is to provide a usable web programming framework, a formal semantics specifying the exact behavior of its main APIs is available http://tzbob.be/gavial/semantics.pdf.

While Gavial has matured, we have plenty of future work in mind. The FFI already allows us to access external tools like databases, but we would prefer to have more APIs native to FRP. We would like to build an FRP database API. We imagine reading from the database using non-discrete behaviors (similar to reading from the DOM) and modifying database state using events.

Acknowledgements Bob Reynders held an SB fellowship of the Research Foundation - Flanders (FWO) during this work. This work has been partly supported by the FWOSBO Tearless project. This research was partly supported by the National Research Foundation of Korea grants from MoE (No. 2019RIIIA3Aoi058608).

\section{References}

[I] Vincent Balat. Client-server Web Applications Widgets. In Proceedings of the 22nd International Conference on World Wide Web, WWW Companion, pages I9-22. Association for Computing Machinery, 20I3. doi:10.1145/2487788.2487795.

[2] Gérard Berry and Georges Gonthier. The Esterel synchronous programming language: Design, semantics, implementation. Science of Computer Programming, I9(2):87-I52, I992. doi:10.1016/0167-6423(92)90005-V.

[3] Gérard Berry and Manuel Serrano. Hop and HipHop: Multitier web orchestration. In Distributed Computing and Internet Technology, volume 8337 of LNCS, pages I-I3. Springer International Publishing, 20I4. doi:10.1007/978-3-319-04483-5_1.

[4] Andoni Lombide Carreton, Stijn Mostinckx, Tom Van Cutsem, and Wolfgang De Meuter. Loosely-coupled distributed reactive programming in mobile ad hoc networks. In International Conference on Modelling Techniques and Tools for Computer Performance Evaluation, volume 6I4I of LNCS, pages 4I-60. Springer, 20Io. doi:10.1007/978-3-642-13953-6_3. 
[5] Adam Chlipala. An optimizing compiler for a purely functional web-application language. In Proceedings of the 2oth ACM SIGPLAN International Conference on Functional Programming, ICFP, pages IO-2I. ACM, 20I5. doi:10.1145/2858949. 2784741.

[6] Adam Chlipala. Ur/Web: A Simple Model for Programming the Web. In Proceedings of the 42nd Annual ACM SIGPLAN-SIGACT Symposium on Principles of Programming Languages, POPL, pages I53-I65. Association for Computing Machinery, 20I5. doi:10.1145/2676726.2677004.

[7] Kwanghoon Choi and Byeong-Mo Chang. A theory of RPC calculi for client-server model. Journal of Functional Programming, 29, 2019. doi:10.1017/ S0956796819000029.

[8] Ezra Cooper, Sam Lindley, Philip Wadler, and Jeremy Yallop. Links: Web programming without tiers. In Formal Methods for Components and Objects, LNCS, pages 266-296. Springer Berlin Heidelberg, 2007. doi:10.1007/978-3-540-74792-5_12.

[9] Evan Czaplicki and Stephen Chong. Asynchronous functional reactive programming for GUIs. In Proceedings of the 34th ACM SIGPLAN Conference on Programming Language Design and Implementation, PLDI, pages 4II-422. Association for Computing Machinery, 2013. doi:10.1145/2491956.2462161.

[Io] Sébastien Doeraene. Scala.js: Type-directed interoperability with dynamically typed languages. Technical report, EPFL, 20I3. URL: http://infoscience.epfl.ch/ record/190834.

[II] Joscha Drechsler, Guido Salvaneschi, Ragnar Mogk, and Mira Mezini. Distributed REScala: An Update Algorithm for Distributed Reactive Programming. In Proceedings of the 20I4 ACM International Conference on Object Oriented Programming Systems Languages \& Applications, OOPSLA, pages 36I-376. Association for Computing Machinery, 20I4. doi:10.1145/2660193.2660240.

[12] Conal Elliott and Paul Hudak. Functional reactive animation. In Proceedings of the second ACM SIGPLAN international conference on Functional programming, ICFP, page 263-273. Association for Computing Machinery, 1997. doi:10.1145/ 258948.258973.

[13] Conal M. Elliott. Push-pull Functional Reactive Programming. In Proceedings of the 2nd ACM SIGPLAN symposium on Haskell, Haskell, pages 25-36. Association for Computing Machinery, 2009. doi:10.1145/258948.258973.

[14] Ingo Maier and Martin Odersky. Higher-Order Reactive Programming with Incremental Lists. In European Conference on Object-Oriented Programming, volume 7920 of $L N C S$, pages 707-73I. Springer International Publishing, 2013. doi:10.1007/978-3-642-39038-8_29.

[15] Geoffrey Mainland, Greg Morrisett, and Matt Welsh. Flask: Staged Functional Programming for Sensor Networks. In Proceedings of the I3th ACM SIGPLAN international conference on Functional programming, ICFP, pages 335-346. ACM, 2008. doi:10.1145/1411204.1411251.

[16] Alessandro Margara and Guido Salvaneschi. We have a DREAM: Distributed reactive programming with consistency guarantees. In Proceedings of the 8th 
ACM International Conference on Distributed Event-Based Systems, DEBS, pages I42-I53. Association for Computing Machinery, 20I4. doi:10.1145/2611286.2611290.

[17] Erik Meijer. Reactive extensions (Rx): curing your asynchronous programming blues. In ACM SIGPLAN Commercial Users of Functional Programming, CUFP, page II. ACM, 20I0. doi:10.1145/1900160.1900173.

[18] Florian Myter, Christophe Scholliers, and Wolfgang De Meuter. Distributed reactive programming for reactive distributed systems. The Art, Science, and Engineering of Programming, 3(3):5:I-5:52, February 2019. arXiv:1902.00524v1, doi:10.22152/programming-journal.org/2019/3/5.

[19] Matthias Neubauer and Peter Thiemann. From sequential programs to multitier applications by program transformation. In Proceedings of the 32nd ACM SIGPLAN-SIGACT symposium on Principles of programming languages, POPL, page 22I-232. Association for Computing Machinery, 2005. doi:10.1145/1047659.1040324.

[20] Martin Odersky, Olivier Blanvillain, Fengyun Liu, Aggelos Biboudis, Heather Miller, and Sandro Stucki. Simplicitly: Foundations and applications of implicit function types. Proceedings of the ACM on Programming Languages, 2(POPL), 20I7. doi:10.1145/3158130.

[2I] Bruno C.d.S. Oliveira, Adriaan Moors, and Martin Odersky. Type classes as objects and implicits. In Proceedings of the ACM international conference on Object oriented programming systems languages and applications, OOPSLA, pages 34I-360. Association for Computing Machinery, 20I0. doi:10.1145/1869459.1869489.

[22] Laure Philips, Coen De Roover, Tom Van Cutsem, and Wolfgang De Meuter. Towards Tierless Web Development without Tierless Languages. In Proceedings of the 2014 ACM International Symposium on New Ideas, New Paradigms, and Reflections on Programming \& Software, Onward!, pages 69-81. Association for Computing Machinery, 20I4. doi:10.1145/2661136.2661146.

[23] Gabriel Radanne, Jérôme Vouillon, and Vincent Balat. Eliom: A core ml language for tierless web programming. In Asian Symposium on Programming Languages and Systems, volume IooI7 of LNCS, pages 377-397. Springer International Publishing, 20I6. doi:10.1007/978-3-319-47958-3_20.

[24] Bob Reynders and Dominique Devriese. Efficient Functional Reactive Programming Through Incremental Behaviors. In Asian Symposium on Programming Languages and Systems, volume I0695 of LNCS, pages 32I-338. Springer International Publishing, 20I7. doi:10.1007/978-3-319-71237-6_16.

[25] Bob Reynders, Dominique Devriese, and Frank Piessens. Multi-tier functional reactive programming for the web. In Proceedings of the 2014 ACM International Symposium on New Ideas, New Paradigms, and Reflections on Programming \& Software, Onward!, page 55-68. Association for Computing Machinery, 2014. doi:10.1145/2661136.2661140.

[26] Bob Reynders, Dominique Devriese, and Frank Piessens. Experience Report: Functional Reactive Programming and the DOM. In Companion to the first International Conference on the Art, Science and Engineering of Programming, 
ProWeb, pages I-6. Association for Computing Machinery, 20I7. doi:10.1145/ 3079368.3079405.

[27] Bob Reynders, Michael Greefs, Dominique Devriese, and Frank Piessens. Scalagna o.I: Towards Multi-tier Programming with Scala and Scala.Js. In Conference Companion of the 2nd International Conference on Art, Science, and Engineering of Programming, ProWeb, pages 69-74. Association for Computing Machinery, 2018. doi:10.1145/3191697.3191731.

[28] Guido Salvaneschi, Joscha Drechsler, and Mira Mezini. Towards distributed reactive programming. In International Conference on Coordination Languages and Models, volume 7890 of LNCS, pages 226-235. Springer, 2013. doi:10.1007/9783-642-38493-6_16.

[29] Manuel Serrano. Programming web multimedia applications with hop. In Proceedings of the I5th ACM international conference on Multimedia, MM, page IOOI-IOO4. Association for Computing Machinery, 2007. doi:10.1145/1291233. 1291450.

[30] Manuel Serrano and Vincent Prunet. A Glimpse of Hopjs. In Proceedings of the 2Ist ACM SIGPLAN International Conference on Functional Programming, ICFP, pages I80-I92. Association for Computing Machinery, 2016. doi:10.1145/2951913.2951916.

[3I] Manuel Serrano and Christian Queinnec. A multi-tier semantics for Hop. HigherOrder and Symbolic Computation, 23(4):409-43I, 2010. doi:10.1007/s10990-0109061-9.

[32] Atze van der Ploeg and Koen Claessen. Practical Principled FRP: Forget the Past, Change the Future, FRPNow! In Proceedings of the 2oth ACM SIGPLAN International Conference on Functional Programming, ICFP, pages 302-3I4. Association for Computing Machinery, 2015. doi:10.1145/2784731.2784752.

[33] Colin Vidal, Gérard Berry, and Manuel Serrano. Hiphop.js: A language to orchestrate web applications. In Proceedings of the 33rd Annual ACM Symposium on Applied Computing, SAC, pages 2I93-2I95, 20I8. doi:10.1145/3167132.3167440.

[34] Tom Murphy Vii, Karl Crary, and Robert Harper. Type-safe distributed programming with ML5. In International Symposium on Trustworthy Global Computing, volume 49I2 of LNCS, pages I08-I23. Springer, 2007. doi:10.1007/978-3-540-786634_9.

[35] Pascal Weisenburger, Mirko Köhler, and Guido Salvaneschi. Distributed system development with ScalaLoci. Proceedings of the ACM on Programming Languages, 2(OOPSLA):I29:I-I29:30, 2018. doi:10.1145/3276499.

[36] Daniel Winograd-Cort and Paul Hudak. Settable and Non-interfering Signal Functions for FRP: How a First-order Switch is More Than Enough. In Proceedings of the I4th ACM SIGPLAN International Conference on Functional Programming, ICFP, pages 213-225. Association for Computing Machinery, 20I4. doi:10.1145/ 2628136.2628140 . 


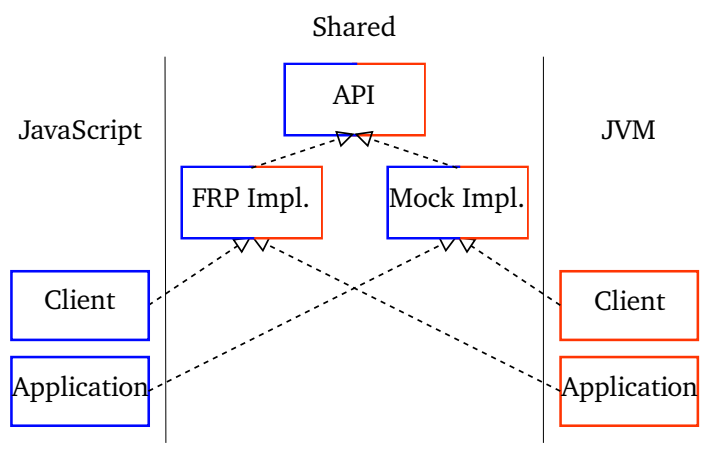

Figure 7 Structure of the Gavial implementation.

\section{A Implementation: Gavial Architecture}

There are several layers of implementation to Gavial as shown in figure 7. There are JavaScript (blue), JVM (red) and shared (blue \& red) sections for their resp. platforms. A shared code section defines code that is included in both platforms.

At the top of Gavial there is an API definition that defines all primitives for a single tier, these include events, behaviors, tier-crossing primitives, etc. This definition has two non-platform specific implementations: FRP and Mock.

An FRP implementation of a tier implements the API with an FRP library. Events and behaviors actually work and cross-tier dependencies are passed through FRP primitives. A Mock implementation on the other hand implements nothing but multitier dependency tracking, the FRP primitives and their operations are essentially null-ops.

Both JVM and JavaScript libraries make use of the Mock and FRP tier. Note that the Client tier should do nothing on the JVM while the Application tier should not do anything in JavaScript. Both these tiers are implemented using the Mock tier while the FRP tier is used to implement the others. 


\section{About the authors}

Bob Reynders is a post doctoral researcher at Chonnam National University. Contact him at tzbobr@gmail.com.

Frank Piessens is a professor in the research group DistriNet (Distributed Systems and Computer Networks) at the Computer Science department of the Katholieke Universiteit Leuven. His main research interests are in the field of software security, where he focuses on the development of high-assurance techniques to deal with implementation-level software vulnerabilities and bugs, including techniques such as software verification, run-time monitoring, type systems, language based security and hardware-software co-design for security.

Dominique Devriese is an assistant professor in the Software Languages Lab at the VUB. His research interests are; formalising properties of object-oriented and object-capability programming languages, formal reasoning about capability machines (CPUs with a built-in form of low-level object capabilities), secure compilation and full abstraction properties and applying logical relations to prove them, and functional and dependently typed programming and programming languages, particularly Agda and Haskell 